\title{
Supporting information: Antifungal susceptibility testing of Aspergillus niger on silicon microwells by intensity-based reflectometric interference spectroscopy
}

Christopher Heuer $\uparrow \perp$, Heidi Leonard $\dagger \perp$, Nadav Nitzan $\dagger$, Ariella Lavy-Alperovitch\$, Naama Massad-Ivanir $†$, Thomas Scheper $\neq$, Ester Segal †*

$\uparrow$ Department of Biotechnology and Food Engineering, § Department of Biology, Technion Israel Institute of Technology, Haifa, 3200003, Israel,

I Institute of Technical Chemistry, Leibniz University Hannover, 30167 Hannover, Germany.

$\perp$ Equal contribution

* Corresponding author, Email: esegal@technion.ac.il 


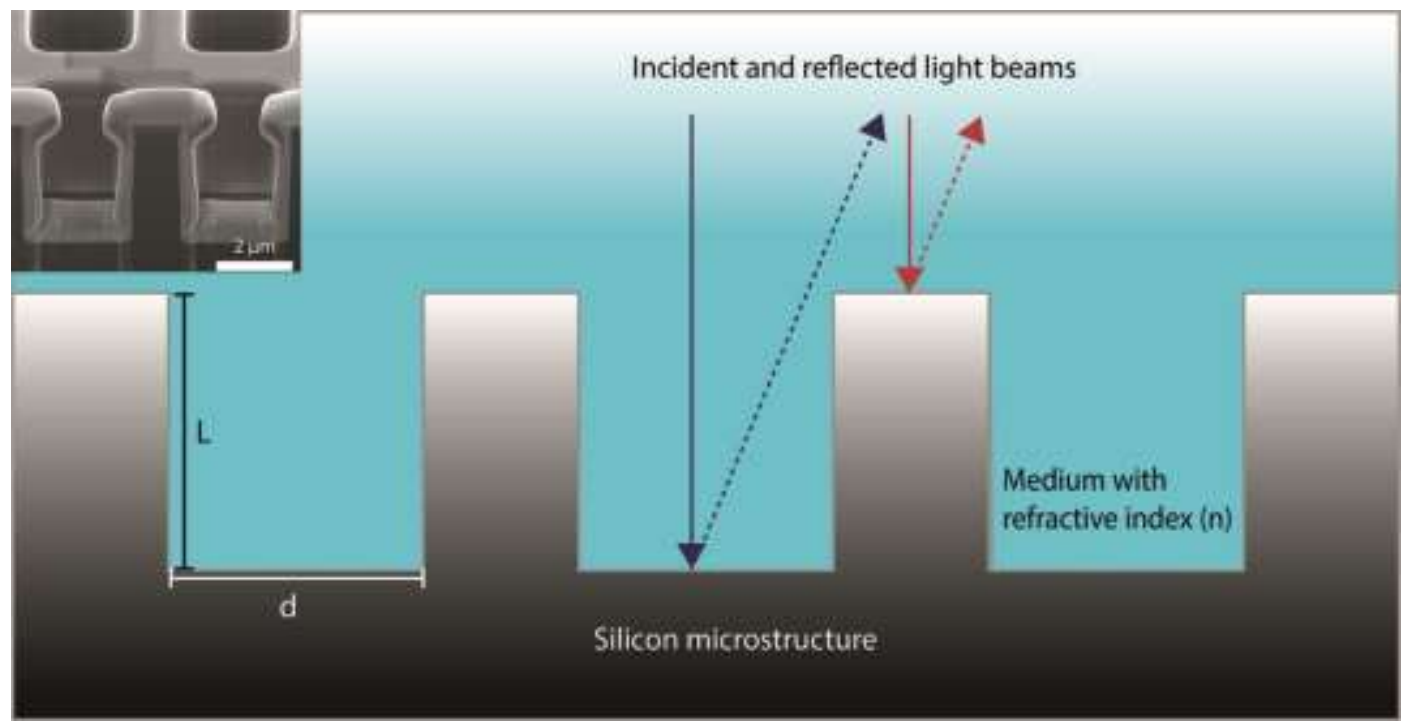

Figure S1. Microstructure and reflectance from of a photonic silicon chip. Schematic illustration of the photonic gratings and a characteristic cross-sectional SEM image (inset). The grating is composed of silicon and the filling medium is typically a conidia suspension with a refractive index of $\mathrm{n}$ (conidia are not shown in this drawing). L represents the height of the microtopology and $d$ is its width. Incident light hits both the bottom of the matrix (blue rays) and the top of the structures (red rays). The optical path difference (OPD) is the product of $2 \mathrm{~L}$ and the refractive index of the filling medium. 


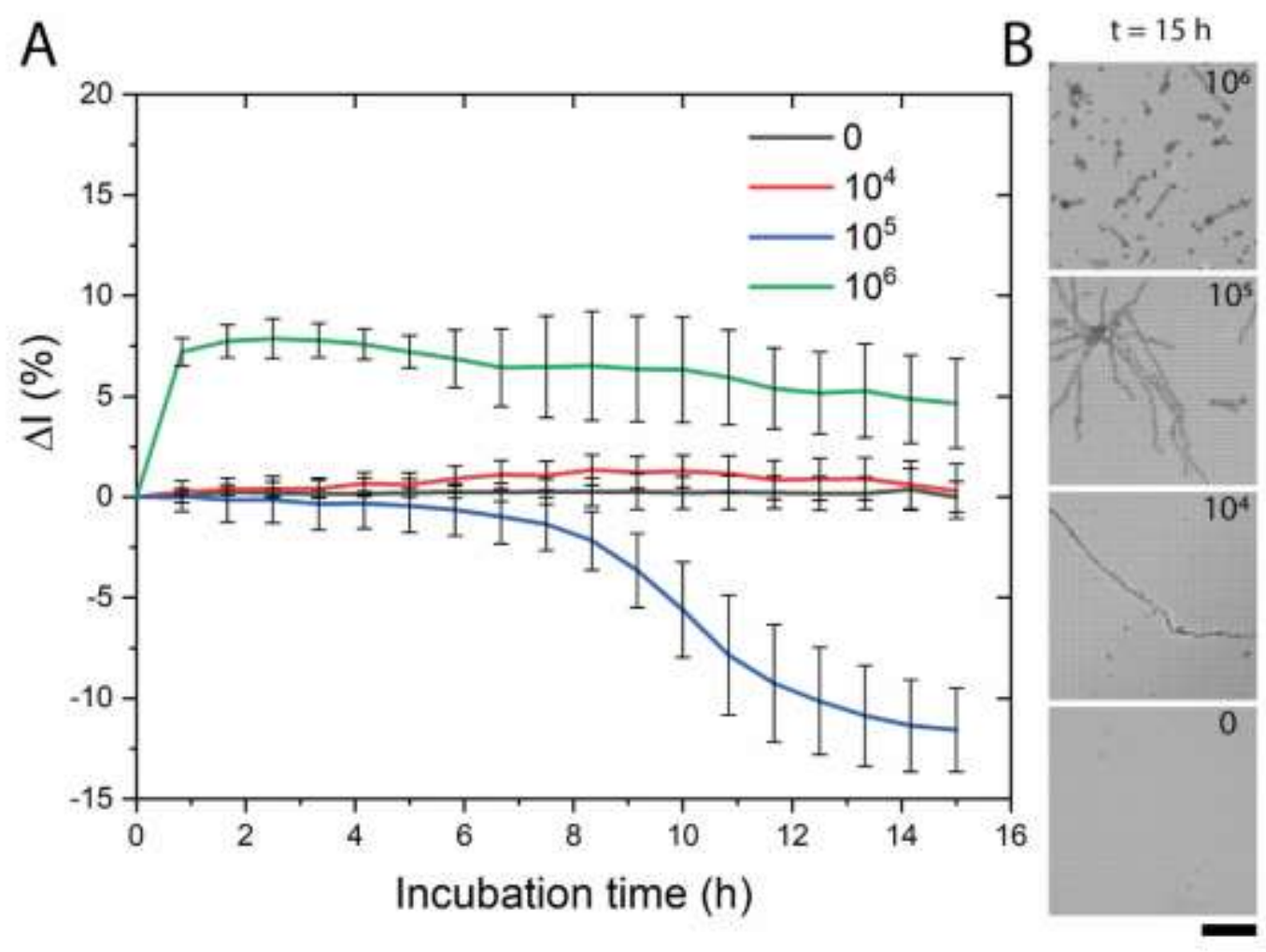

Figure S2. iPRISM for monitoring the growth of $A$. niger at different initial conidia concentrations. (A) $\Delta I(\%)$ over time for conidial seeding suspensions of $0,10^{4}, 10^{5}$ and $10^{6}$ conidia $\mathrm{mL}^{-1}$; Average and standard deviation for triplicates $(\mathrm{n}=3)$ were calculated every $50 \mathrm{~min}$. (B) Optical microscope images of $A$. niger fixed after $15 \mathrm{~h}$ of incubation with conidial seeding suspensions of $0,10^{4}, 10^{5}$ and $10^{6}$ conidia $\mathrm{mL}^{-1}$. Black scale bar represents $50 \mu \mathrm{m}$. 


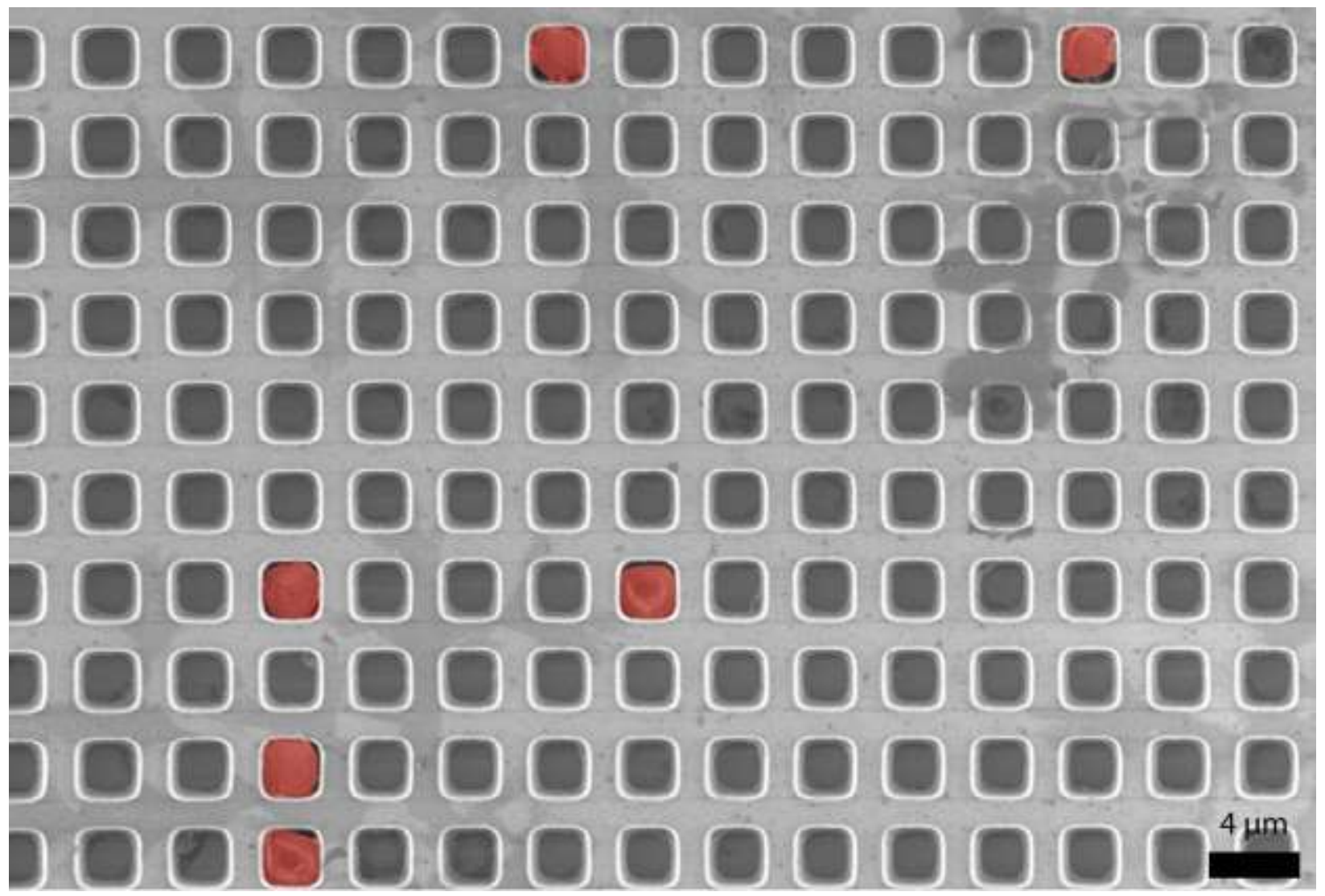

Figure S3. The proportion of conidia seeded per well. A representative HR-SEM image with six conidia (false-colored for clarity) confined within a total of 150 microwells. A typical proportion of conidia seeded per well is $2-5$ conidia per 100 microwells. 

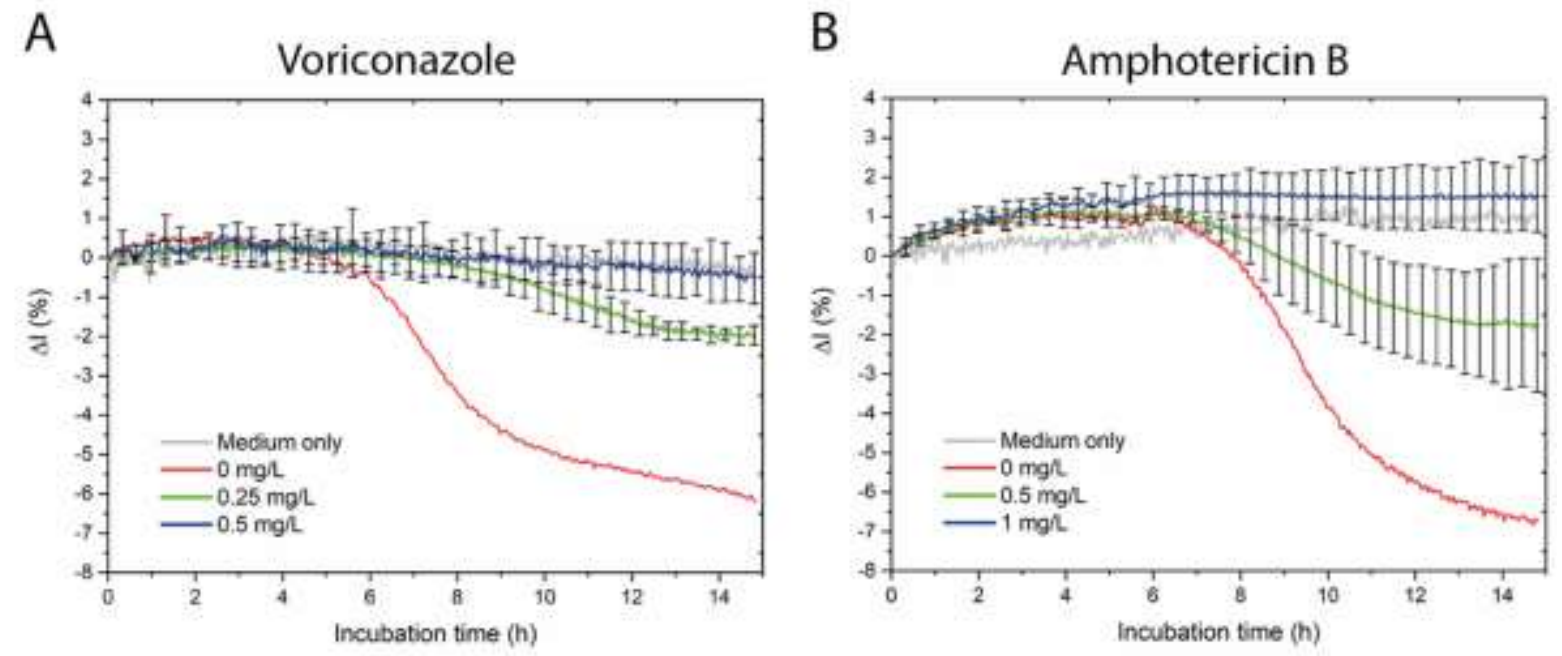

Figure S4. Comparison of the MIC and the highest subinhibitory concentration of voriconazole and amphotericin B against Aspergillus niger in triplicate $(\mathrm{n}=3)$. (A) Comparison of the MIC $\left(0.5 \mathrm{mg} \mathrm{L}^{-1}\right)$ and highest subinhibitory concentration $\left(0.25 \mathrm{mg} \mathrm{L}^{-1}\right)$ of voriconazole against $A$. niger according to Figure 3A. (B) Comparison of MIC (1 mg L $\left.\mathrm{L}^{-1}\right)$ and highest subinhibitory concentration $\left(0.5 \mathrm{mg} \mathrm{L}^{-1}\right)$ of amphotericin $\mathrm{B}$ against $A$. niger according to Figure $3 \mathrm{~B}$. T-tests were performed for both drugs after 8,10 and $12 \mathrm{~h}$ of incubation and in the end of the assay $(\sim 15 \mathrm{~h})$, showing that the intensity signal for the MIC and the highest subinhibitory concentration are significantly different $(\mathrm{p}<0.05)$ for voriconazole and amphotericin B after 12 and $10 \mathrm{~h}$, respectively. 
The ZPrime Score (Z'), based on Zhang et al. ${ }^{1}$, is calculated using the following equation:

$$
Z^{\prime}=1-\frac{3 *\left(\sigma_{\text {MIC }}+\sigma_{\text {sub MIC }}\right)}{\left|\mu_{\text {MIC }}-\mu_{\text {sub MIC }}\right|}
$$

$\sigma_{\text {MIC }}$ and $\sigma_{\text {sub MIC }}$ are the standard deviations of the MIC and the first subinhibitory concentration. $\mu_{\text {MIC }}$ and $\mu_{\text {sub MIC }}$ represent the mean $(n=3)$ of the MIC and the first subinhibitory concentration. The calculated values for voriconazole and amphotericin B are presented in Table S1 using the data from Figure S4.

Table S1. ZPrime Score after $10 \mathrm{~h}, 12 \mathrm{~h}$ and in the end of the assay $(\sim 15 \mathrm{~h})$ for voriconazole and amphotericin B.

\begin{tabular}{|l|l|l|}
\hline Time (h) & $\begin{array}{l}\text { ZPrime Score } \\
\text { voriconazole }\end{array}$ & $\begin{array}{l}\text { ZPrime Score } \\
\text { amphotericin B }\end{array}$ \\
\hline $\mathbf{1 0}$ & -3.45 & -1.4 \\
\hline $\mathbf{1 2}$ & -0.89 & -0.99 \\
\hline $\mathbf{1 5}$ & -0.52 & -1.37 \\
\hline
\end{tabular}


Table S2. Sequencing result for the forward primer ITSF1.

\begin{tabular}{|l|}
\hline DNA sequence \\
\hline 5' \\
TTCCGTAGGTGAACCTGCGGNGGATCATTACCGAGTGCGGGTCCTTTGGGCCCAACC \\
TCCCATCCGTG \\
TCTATTGTACCCTGTTGCTTCGGCGGGCCCGCCGCTTGTCGGCCGCCGGGGGGGCGC \\
CTCTGCCCCCCGGGCCCGTGCCC \\
GCCGGAGACCCCAACACGAACACTGTCTGAAAGCGTGCAGTCTGAGTTGATTGAAT \\
GCAATCAGTTAAAACTTTCAACAA \\
TGGATCTCTTGGTTCCGGCATCGATGAAGAACGCAGCGAAATGCGATAACTAATGTG \\
AATTGCAGAATTCAGTGAATCAT \\
CGAGTCTTTGAACGCACATTGCGCCCCCTGGTATTCCGGGGGGCATGCCTGTCCGAG \\
CGTCATTGCTGCCCTCAAGCCCG \\
GCTTGTGTGTTGGGTCGCCGTCCCCCTCTCCGGGGGGACGGGCCCGAAAGGCAGCG \\
GCGGCACCGCGTCCGATCCTCGAG \\
CGTATGGGGCTTTGTCACATGCTCTGTAGGATTGGCCGGCGCCTGCCGACGTTTTCC \\
AACCATTCTTTCCAGGTTGACCT \\
CGGATCAGGTAGGGATACCCGCTGAACTTAAGCATATCAATAAGCGGGAGGAA - 3'
\end{tabular}

Protocol for species identification by Internal transcribed spacer (ITS) regions:

DNA extraction. $A$. niger isolate $\mathrm{HCN} 18$ was grown overnight at room temperature in liquid potato dextrose yeast extract casein hydrolysate medium (PDYC, $24 \mathrm{~g} \mathrm{~L}^{-1}$ potato dextrose broth, $2 \mathrm{~g} \mathrm{~L}^{-1}$ yeast extract and $1.2 \mathrm{~g} \mathrm{~L}^{-1}$ casein hydrolysate, all Difco, USA) in a petri dish without shaking. For DNA extraction two full spatulas of the mycelium were transferred to a clean tube with $100 \mu \mathrm{L}$ Zirconia/Silica beads (Biospec Products, Inc., USA) and $500 \mu \mathrm{L}$ DNA extraction buffer (200 mM tris(hydroxymethyl)aminomethane hydrochloride (Tris HCl, Sigma Aldrich, Israel), $0,5 \%$ sodium dodecyl sulfate (SDS, Thermo Scientific, USA), $25 \mathrm{mM}$ ethylenediaminetetraacetic acid (EDTA, Sigma Aldrich, Israel), $250 \mathrm{mM} \mathrm{NaCl}$ (Merck, 
Germany) and mixed for one minute on a vortex mixer (Scientific Industries, Inc., USA). The sample was incubated for $30 \mathrm{~min}$ at room temperature and centrifuged for $10 \mathrm{~min}$ at $4{ }^{\circ} \mathrm{C}$ and $12500 \mathrm{rpm}$ (Centrifuge 5417R, Eppendorf AG, Germany). The supernatant was transferred to a clean tube, and an equal volume of isopropanol was added. The sample was incubated for 20 minutes at $-20{ }^{\circ} \mathrm{C}$ and afterward centrifuged at $13000 \mathrm{rpm}$ for $2 \mathrm{~min}$. Subsequently, the supernatant was discarded, and the resulting pellet was washed with $1 \mathrm{~mL} 70 \%$ ethanol followed by centrifugation at $13000 \mathrm{rpm}$ for $2 \mathrm{~min}$. The supernatant was rejected again, and the pellet was left for drying for around $1 \mathrm{~h}$ and resuspended in $50 \mu \mathrm{L}$ of sterile double distilled water. The concentration of the genomic DNA was determined by using a Nanodrop one (Thermo Scientific, USA) resulting in a concentration of $56.4 \mathrm{ng} / \mu \mathrm{L}$ with an A260:A280 ratio of 1.85.

PCR Amplification of ITS. Species identification of isolate HCN 18 was carried out by sequencing the ITS1 and ITS2 internal transcribed spacer regions by using the primers ITS1F (5'-CTTGGTCATTTAGAGGAAGTAA-3') and ITS4 (5'-TCCTCCGCTTATTGATATGC-3'). ${ }^{2}$ PCR amplification reaction mixtures contained 1 x PCR buffer, $0.2 \mathrm{mM}$ premixed dNTP's, 1.25 U of Takara Taq ${ }^{\mathrm{TM}}$ Polymerase (all Takara, Japan), $1 \mu \mathrm{M}$ ITS1F and ITS4 primers and $50 \mathrm{ng}$ genomic DNA in a total volume of $25 \mu \mathrm{L}$. For non-template control the PCR reaction mixture contained all the components as mentioned above but no genomic Aspergillus DNA. PCR amplification was carried out in a biometra TGradient Thermocycler (Analytik Jena, Germany) using an initial denaturation step at $94{ }^{\circ} \mathrm{C}$ for $2 \mathrm{~min}$, followed by 29 cycles at $94{ }^{\circ} \mathrm{C}$ for $30 \mathrm{~s}, 55$ ${ }^{\circ} \mathrm{C}$ for $30 \mathrm{~s}$ and $72{ }^{\circ} \mathrm{C}$ for $30 \mathrm{~s}$, with a final extension for $2 \mathrm{~min}$ at $72{ }^{\circ} \mathrm{C}$. Successful amplification was confirmed after standard electrophoresis in a 1\% agarose gel by visualizing the PCR product under UV light by using the SimplySafe ${ }^{\mathrm{TM}}$ dye (EURx, Poland). The PCR fragment was extracted from the gel by using a DNA gel extraction kit (AccuPrep Gel Purification Kit, 
Bioneer, South Korea) and sent to Hylabs (Israel) for sequencing by using an ABI 3730xl DNA Analyzer (Thermo Scientific, USA). Sequence alignment was done using the nucleotide nucleotide BLAST search algorithm BLASTn, at the NCBI website (https://blast.ncbi.nlm.nih.gov/Blast.cgi). Species was identified as Aspergillus niger (NCBI Accession number: MK182796.1).

\section{REFERENCES}

(1) Zhang; Chung; Oldenburg. A Simple Statistical Parameter for Use in Evaluation and Validation of High Throughput Screening Assays. Journal of biomolecular screening 1999, 4, $67-73$

(2) White, T. J.; Bruns, T.; Lee, S.; Taylor, J. Amplification and Direct Sequencing of Fungal Ribosomal RNA Genes for Phylogenetics. PCR Protoc. Guide Methods Appl. 1990, 18 (1), 315322. 